\title{
Normalization of exact quasiparticle wave functions in the Green's function method guaranteed by the Ward identity
}

\author{
Takeru Nakashima, Hannes Raebiger $\odot$, and Kaoru Ohno ๑* \\ Department of Physics, Yokohama National University, 79-5 Tokiwadai, Hodogaya-ku, Yokohama 240-8501, Japan
}

(Received 10 August 2020; revised 7 October 2020; accepted 8 November 2021; published 29 November 2021)

\begin{abstract}
It has been a long-standing problem in $N$-electron systems as to how to solve the Dyson equation with the quasiparticle wave functions defined as $\left\langle\Psi_{0}^{N}\left|\psi_{s}(\boldsymbol{r})\right| \Psi_{v}^{N+1}\right\rangle$ and $\left\langle\Psi_{\mu}^{N-1}\left|\psi_{s}(\boldsymbol{r})\right| \Psi_{0}^{N}\right\rangle$, which are not mutually orthogonal and have a norm less than 1 . We show that quasiparticle wave functions without multiple excitations can exactly be normalized to unity owing to the Ward identity and the vertex function in $\left(\boldsymbol{q}, \omega^{\prime}-\omega\right) \rightarrow 0$, although they are not necessarily mutually orthogonal. Since they satisfy an eigenvalue equation with the hermitized self-energy due to Baym-Kadanoff's conservation laws, the present theory can be regarded as an extension of the Kohn-Sham density functional theory, with correlated, interacting, one-electron orbitals.
\end{abstract}

DOI: 10.1103/PhysRevB.104.L201116

The quasiparticle (QP) approach such as the GW approximation (GWA) [1-13] and the GW $\Gamma$ approach [14-16] in the Green's function method in many-body perturbation theory (MBPT) has been used in the study of optical properties and electronic structures of materials. The QPs mean oneelectron difference between the initial $N$-electron ground state $\left|\Psi_{0}^{N}\right\rangle$ (satisfying $\left.H\left|\Psi_{0}^{N}\right\rangle=E_{0}^{N}\left|\Psi_{0}^{N}\right\rangle\right)$ and the final $(N \pm$ 1)-electron excited states $\left|\Psi_{\lambda}^{N \pm 1}\right\rangle$ (satisfying $H\left|\Psi_{\lambda}^{N \pm 1}\right\rangle=$ $E_{\lambda}^{N \pm 1}\left|\Psi_{\lambda}^{N \pm 1}\right\rangle$ ). ( $H$ is the Hamiltonian of an inhomogeneous system.) The QP energies, which are defined as $\varepsilon_{\mu}=E_{0}^{N}-$ $E_{\mu}^{N-1}$ for an occupied (occ) $\mu$ level and $\varepsilon_{v}=E_{v}^{N+1}-E_{0}^{N}$ for an empty (emp) $v$ level, directly represent the photoemission (PE) and inverse photoemission (IPE) energies, and the QP wave functions (QPWFs), which are defined as $\phi_{\mu}(\boldsymbol{r}, s)=\left\langle\Psi_{\mu}^{N-1}\left|\psi_{s}(\boldsymbol{r})\right| \Psi_{0}^{N}\right\rangle$ for $\mu \in$ occ and $\phi_{v}(\boldsymbol{r}, s)=$ $\left\langle\Psi_{0}^{N}\left|\psi_{s}(\boldsymbol{r})\right| \Psi_{v}^{N+1}\right\rangle$ for $v \in \mathrm{emp}$ ( $s$ is the spin coordinate), describe the created hole and electron (particle) distribution. Sandwiching the anticommutation relation of the annihilation and creation operators $\left\{\psi_{s}\left(\boldsymbol{r}_{1}\right), \psi_{s^{\prime}}^{\dagger}\left(\boldsymbol{r}_{2}\right)\right\}$ with $\left\langle\Psi_{0}^{N}|,| \Psi_{0}^{N}\right\rangle$ and inserting the complete sets of the $(N \pm 1)$-electron intermediate states inside the bracket, one derives the completeness condition for the QPWFs [17]:

$$
\sum_{\lambda \in \text { all }} \phi_{\lambda}\left(\boldsymbol{r}_{1}, s\right) \phi_{\lambda}^{*}\left(\boldsymbol{r}_{2}, s^{\prime}\right)=\delta_{s s^{\prime}} \delta\left(\boldsymbol{r}_{1}-\boldsymbol{r}_{2}\right) .
$$

However, the QPWFs are not orthogonal to each other, not linearly independent [17], and their norm is less than 1 [18-20]; see Eq. (21) below. There are an infinite number of the occ QPWFs $\phi_{\mu}(\boldsymbol{r}, s)$ even for finite $N$-electron systems, as there

\footnotetext{
*ohno@ynu.ac.jp
}

Published by the American Physical Society under the terms of the Creative Commons Attribution 4.0 International license. Further distribution of this work must maintain attribution to the author(s) and the published article's title, journal citation, and DOI. are infinite numbers of $(N-1)$-electron states $\left|\Psi_{\mu}^{N-1}\right\rangle$. This implies that the electron spin density $[2,17]$

$$
\rho_{s}(\boldsymbol{r})=\left\langle\Psi_{0}^{N}\left|\psi_{s}^{\dagger}(\boldsymbol{r}) \psi_{s}(\boldsymbol{r})\right| \Psi_{0}^{N}\right\rangle=\sum_{\mu \in \mathrm{occ}}\left|\phi_{\mu}(\boldsymbol{r}, s)\right|^{2}
$$

must be expressed with an infinite number of the occ QPWFs, which makes rigorous analyses very difficult. How to solve the Dyson equation by means of these QPWFs [20] has been a long-standing problem. Although this problem has often been bypassed by approximating the self-energy form [8] or by linearizing the self-energy $[9,12,16]$ in the self-consistent (sc) $\mathrm{GW}(\Gamma)$ approaches, the essential problem on the norm of the QPWFs has not been solved yet.

There are also a few other but related problems in the GWA. The first is that the GWA does not correctly satisfy Pauli's exclusion principle, i.e., the self-interaction error remains due to incomplete cancellation between the screened exchange and Hartree terms [21]. The second is that it violates the Ward-Takahashi identity [22-24], which guarantees the gauge invariance, i.e., the local charge conservation. To the best of our knowledge, no exact QP formulation has been given in a first-principles framework yet. The problems are also related to the foundation of density functional theory (DFT) [25] and the Kohn-Sham (KS) theory [26]. Concerning the relation between the KS potential and the self-energy, there is a formula proposed by Sham and Schlüter [27], but our approach claims their exact equivalence in a hermitized form.

The purpose of the present Letter is to solve all these problems in an exact manner. We first assume that, in $N$-electron systems, the two-time spin-density matrix, which satisfies an equation similar to the Dyson equation, is expressed in a bilinear form by $N$ normalized QPWFs $\bar{\phi}_{\lambda}(\boldsymbol{r}, t, s)$. We show that they satisfy exactly the same QP equation, and the problem is reduced to a simple normalization of the QPWFs. The procedure is similar to that of the fully sc GWA [5,11], but the QP energies are complex numbers reflecting a finite lifetime [10]. 
Then, a vast number of irrelevant $(N \pm 1)$-electron states, which are related to multiple excitations, are eliminated from the QP states. We note that the normalization corresponds to taking into account the Ward identity and the vertex correction in the $\left(\boldsymbol{q}, \omega^{\prime}-\omega\right) \rightarrow 0$ limit. Finally, we show that the renormalization can be performed in an exact formulation using the Bethe-Salpeter equation for the vertex function. Since the nonorthogonal but normalized QPWFs and the real part of the QP energies are determined with the hermitized self-energy due to Baym-Kadanoff's conservation laws [24,28] and the electron spin density $\rho_{s}(\boldsymbol{r})$ is exactly expressed by $N$ QPWFs, the present theory can be regarded as an extension of the KS theory.

One-particle Green's function and QP equations. The oneparticle Green's function is defined by $[1,2,17,24,28]$

$$
G_{s}(1,2)=-i\left\langle\Psi_{0}^{N}\left|T\left[\psi_{s}(1) \psi_{s}^{\dagger}(2)\right]\right| \Psi_{0}^{N}\right\rangle,
$$

where numbers are abbreviations for position and time coordinates, i.e., $i=\left(\boldsymbol{r}_{i}, t_{i}\right), \psi_{s}(i)$ are the Heisenberg operators, and $T$ is Wick's time-ordered product. It is expanded as $[1,2,17]$

$$
\begin{aligned}
G_{s}(1,2)= & -i \sum_{\nu \in \mathrm{emp}} \phi_{\nu}(1, s) \phi_{\nu}^{*}(2, s) \theta\left(t_{1}-t_{2}\right) \\
& +i \sum_{\mu \in \mathrm{occ}} \phi_{\mu}^{*}(2, s) \phi_{\mu}(1, s) \theta\left(t_{2}-t_{1}\right),
\end{aligned}
$$

where $\theta\left(t_{1}-t_{2}\right)$ is Heaviside step function, $\phi_{v}(i, s)$ and $\phi_{\mu}(i, s)$ are the time-dependent emp and occ QPWFs [17]:

$$
\phi_{\lambda}(i, s)=\phi_{\lambda}\left(\boldsymbol{r}_{i}, s\right) e^{-i \varepsilon_{\lambda} t_{i}}(\lambda=v \text { or } \mu) .
$$

Here, $\phi_{\lambda}\left(\boldsymbol{r}_{i}, s\right)$ and $\varepsilon_{\lambda}$ are the (time-independent) QPWFs and the QP energies. Note that $\varepsilon_{\lambda}$ become complex numbers in MBPT. By the Fourier transformation, we have $[1,2,17]$

$$
G_{s}\left(\boldsymbol{r}_{1}, \boldsymbol{r}_{2} ; \omega\right)=\sum_{\lambda \in \text { all }} \frac{\phi_{\lambda}\left(\boldsymbol{r}_{1}, s\right) \phi_{\lambda}^{*}\left(\boldsymbol{r}_{2}, s\right)}{\omega-\varepsilon_{\lambda}-i \delta_{\lambda}},
$$

where $\delta_{\lambda}$ are $0^{+}$and $-0^{+}$, respectively, for occ and emp QP states $\left(0^{+}\right.$is a positive infinitesimal number). Therefore the same problem of the QPWFs appears in the Green's function approach.

From Heisenberg's equation of motion for $\psi_{s}(1)$, the oneparticle Green's function satisfies $[1,2,24,28]$

$$
\begin{aligned}
& i \partial_{t_{1}} G_{s}(1,2)-h_{s}\left(\boldsymbol{r}_{1}\right) G_{s}(1,2) \\
& \quad+i \sum_{s^{\prime}} \int d 3 V\left(3,1^{+}\right) K_{s s^{\prime} ; s s^{\prime}}\left(1,3 ; 2,3^{+}\right)=\delta(1,2),
\end{aligned}
$$

where $h_{s}\left(\boldsymbol{r}_{1}\right)=-\frac{1}{2} \nabla_{1}^{2}+v_{s}\left(\boldsymbol{r}_{1}\right)$ represents the one-body part of the Hamiltonian, $V(3,1)=\delta\left(t_{3}-t_{1}\right) /\left|\boldsymbol{r}_{3}-\boldsymbol{r}_{1}\right|$, $i^{+}$denotes $\left(\boldsymbol{r}_{i}, t_{i}+0^{+}\right)$, and $K_{s s^{\prime}}$ is the two-particle Green's function defined by $K_{s s^{\prime} ; s s^{\prime}}\left(1,2 ; 1^{\prime}, 2^{\prime}\right)=$ $-\left\langle\Psi_{0}^{N}\left|T\left[\psi_{s}(1) \psi_{s^{\prime}}(2) \psi_{s^{\prime}}^{\dagger}\left(2^{\prime}\right) \psi_{s}^{\dagger}\left(1^{\prime}\right)\right]\right| \Psi_{0}^{N}\right\rangle$. The last term in the left-hand side of Eq. (7) can be rewritten by introducing the self-energy $\Sigma_{s}(1,3)$ as $[1,2,24,28]$

$$
\begin{aligned}
& i \partial_{t_{1}} G_{s}(1,2)-h_{s}\left(\boldsymbol{r}_{1}\right) G_{s}(1,2)-\int d 3 \Sigma_{s}(1,3) G_{s}(3,2) \\
& =\delta(1,2) .
\end{aligned}
$$

This is the Dyson equation in a differential form. Time Fourier transformation of Eq. (8), $\omega G_{s}\left(\boldsymbol{r}_{1}, \boldsymbol{r}_{2} ; \omega\right)-h_{s}\left(\boldsymbol{r}_{1}\right)$ $G_{s}\left(\boldsymbol{r}_{1}, \boldsymbol{r}_{2} ; \omega\right)-\int d \boldsymbol{r}_{3} \Sigma_{s}\left(\boldsymbol{r}_{1}, \boldsymbol{r}_{3} ; \omega\right) G_{s}\left(\boldsymbol{r}_{3}, \boldsymbol{r}_{2} ; \omega\right)=\delta\left(\boldsymbol{r}_{1}-\boldsymbol{r}_{2}\right)$, yields via the integral around each pole at $\varepsilon_{\lambda}+i \delta_{\lambda}$ with (6) [1-3,29],

$\varepsilon_{\lambda} \phi_{\lambda}\left(\boldsymbol{r}_{1}, s\right)=h_{s}\left(\boldsymbol{r}_{1}\right) \phi_{\lambda}\left(\boldsymbol{r}_{1}, s\right)+\int d \boldsymbol{r}_{2} \Sigma_{s}\left(\boldsymbol{r}_{1}, \boldsymbol{r}_{2} ; \varepsilon_{\lambda}\right) \phi_{\lambda}\left(\boldsymbol{r}_{2}, s\right)$.

This is the QP equation. Note that the self-energy is energy dependent. The solutions of this equation are not orthogonal to each other and have the norm between 0 and 1 [18-20].

Here we propose to solve this problem as follows. Multiplying both sides of (9) by $e^{-i \varepsilon_{\lambda} t_{1}}$ and using the original definition $\Sigma_{s}\left(r_{1}, r_{2} ; \varepsilon_{\lambda}\right)=\int d\left(t_{1}-t_{2}\right) \Sigma_{s}(1,2) e^{i \varepsilon_{\lambda}\left(t_{1}-t_{2}\right)}$, we obtain the time-dependent QP equation $[2,30]$

$$
i \partial_{t_{1}} \phi_{\lambda}(1, s)=h_{s}\left(\boldsymbol{r}_{1}\right) \phi_{\lambda}(1, s)+\int d 2 \Sigma_{s}(1,2) \phi_{\lambda}(2, s) .
$$

Now we define the two-time spin-density matrix $D_{s}^{(-)}(1,2)$ and its complementary function $D_{s}^{(+)}(1,2)$ as

$$
\begin{aligned}
D_{s}^{(-)}(1,2) & \equiv\left\langle\Psi_{0}^{N}\left|\psi_{s}^{\dagger}(2) \psi_{s}(1)\right| \Psi_{0}^{N}\right\rangle \\
& =\sum_{\mu \in \mathrm{occ}} \phi_{\mu}(1, s) \phi_{\mu}^{*}(2, s), \\
D_{s}^{(+)}(1,2) & \equiv\left\langle\Psi_{0}^{N}\left|\psi_{s}(1) \psi_{s}^{\dagger}(2)\right| \Psi_{0}^{N}\right\rangle \\
& =\sum_{\nu \in \mathrm{emp}} \phi_{\nu}(1, s) \phi_{v}^{*}(2, s),
\end{aligned}
$$

which are equal to $\mp i G_{s}(1,2)$ for $t_{1} \lessgtr t_{2}$ and satisfy an equation that is similar to Eq. (8) and readily derived from (10),

$$
i \partial_{t_{1}} D_{s}^{( \pm)}(1,2)=h_{s}\left(\boldsymbol{r}_{1}\right) D_{s}^{( \pm)}(1,2)+\int d 3 \Sigma_{s}(1,3) D_{s}^{( \pm)}(3,2) .
$$

Next, we assume that these functions are expressed by normalized QPWFs $\bar{\phi}_{i}(1, s)(i=1,2, \ldots, N, N+1, N+2, \ldots)$ as

$$
\begin{aligned}
& D_{s}^{(-)}(1,2)=\sum_{i=1}^{N} \bar{\phi}_{i}(1, s) \bar{\phi}_{i}^{*}(2, s), \\
& D_{s}^{(+)}(1,2)=\sum_{i=N+1}^{\infty} \bar{\phi}_{i}(1, s) \bar{\phi}_{i}^{*}(2, s) .
\end{aligned}
$$

Then the necessary condition for the electron spin density $\sum_{s} \int d \boldsymbol{r}_{1} \rho_{s}\left(\boldsymbol{r}_{1}\right)=\sum_{s} \int d \boldsymbol{r}_{1} D_{s}^{(-)}(1,1)=N$ and the completeness condition (1) are automatically satisfied. At each time $t_{1}$, we can introduce the dual orbitals $\widetilde{\phi}_{j}(1, s)(j=$ $1,2, \ldots, N, N+1, N+2, \ldots)$, which satisfy the biorthogonality condition [31],

$$
\int \bar{\phi}_{i}^{*}(1, s) \widetilde{\phi}_{j}(1, s) d \boldsymbol{r}_{1}=\delta_{i j} \quad(i, j \leqslant N \text { or } i, j>N),
$$

and derive $\int D_{s}^{(\mp)}(1,2) \widetilde{\phi}_{i}(2, s) d \boldsymbol{r}_{2}=\bar{\phi}_{i}(1, s)(\mp$ for $i \lesseqgtr N)$. Using this, we readily find that Eq. (12) yields the equation

$$
i \partial_{t_{1}} \bar{\phi}_{i}(1, s)=h_{s}\left(\boldsymbol{r}_{1}\right) \bar{\phi}_{i}(1, s)+\int d 2 \Sigma_{s}(1,2) \bar{\phi}_{i}(2, s) .
$$


(a)

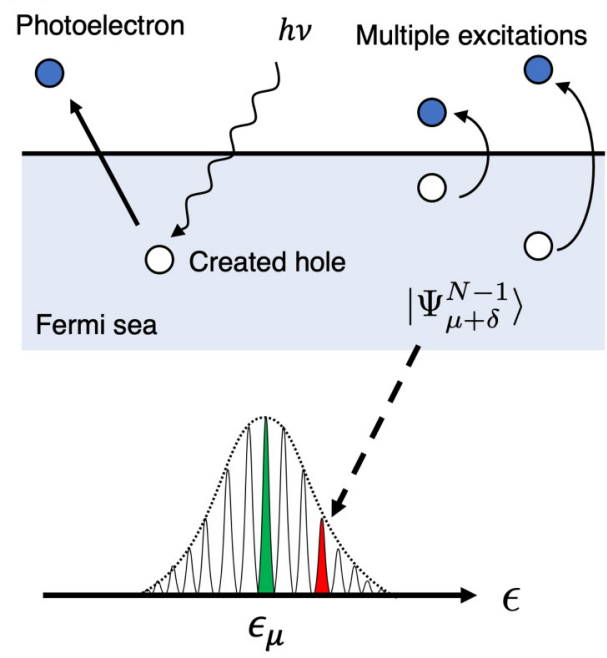

(b)

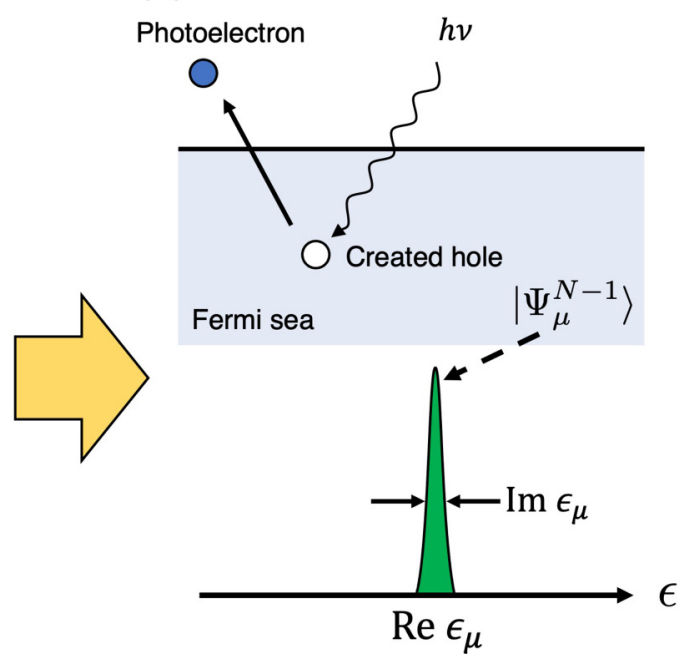

FIG. 1. Illustration of (a) infinite number of $(N-1)$-electron eigenstates $\left|\Psi_{\mu+\delta}^{N-1}\right\rangle$ with multiple excitations around the created hole and (b) the relevant $(N-1)$-electron state $\left|\Psi_{\mu}^{N-1}\right\rangle$ with the created hole.

Equations (10) and (15) have exactly the same form, and thus we conclude that $\bar{\phi}_{i}(1, s)$ is equivalent to the QPWF $\phi_{\mu}(1, s)$ except for a normalization factor. Therefore the electron spin density $\rho_{s}\left(\boldsymbol{r}_{1}\right)=D_{s}^{(-)}(1,1)$ is expressed by $N$-normalized QPWFs except for normalization. Those functions are not necessarily mutually orthogonal. Here is a hint to solve the problem. There are a lot of independent excitations that are not directly associated with the PE and IPE processes in the $(N \pm 1)$-electron states $\left|\Psi_{\lambda}^{N \pm 1}\right\rangle$; see Fig. 1(a). However, they can be eliminated in the summation over all occupied and empty states in Eqs. (1), (2), (4), (6), and (11), and instead we accept that the QP energies have an imaginary part, which represents the peak width and the inverse of the QP lifetime; see Fig. 1(b). Then each hole (electron) QP state associated with one final PE $\left|\Psi_{\mu}^{N-1}\right\rangle\left(\operatorname{IPE}\left|\Psi_{v}^{N+1}\right\rangle\right)$ state, which does not include any multiple excitations or Auger-like process involving the recombination and the second excitations, etc., may have a norm equal to the amount of one electron exactly.

Normalization procedure. For convenience, we introduce an abstract representation $\left|\phi_{\lambda}\right\rangle$ by $\left\langle\boldsymbol{r}, s \mid \phi_{\lambda}\right\rangle=\phi_{\lambda}(\boldsymbol{r}, s) \quad(\lambda=$ $v$ or $\mu$ ) and write the Green's function as $G_{s}\left(\boldsymbol{r}_{1}, \boldsymbol{r}_{2} ; \omega\right) \delta_{s s^{\prime}}=$ $\left\langle\boldsymbol{r}_{1}, s|G(\omega)| \boldsymbol{r}_{2}, s^{\prime}\right\rangle$. (Although it is assumed for simplicity that the Green's function is diagonal in spin, it is straightforward to extend the present theory to a more general case where the Green's function is not diagonal in spin, for example, by the spin-orbit interaction.) Here $G(\omega)$ is expressed as

$$
G(\omega)=\sum_{\lambda \in \text { all }} \frac{\left|\phi_{\lambda}\right\rangle\left\langle\phi_{\lambda}\right|}{\omega-\varepsilon_{\lambda}-i \delta_{\lambda}}
$$

The one-body term and the self-energy are written in similar operator forms $h$ and $\Sigma(\omega)$. Under Baym-Kadanoff's conservation laws [24,28], the Dyson equation is expressed as [32]

$$
[\omega-h-\Sigma(\omega)] G(\omega)=G(\omega)[\omega-h-\Sigma(\omega)]=1 .
$$

The integral around each pole of $G(\omega)$ with (16) yields

$$
\left[\varepsilon_{\lambda}-h-\Sigma\left(\varepsilon_{\lambda}\right)\right]\left|\phi_{\lambda}\right\rangle=\left\langle\phi_{\lambda}\right|\left[\varepsilon_{\lambda}-h-\Sigma\left(\varepsilon_{\lambda}\right)\right]=0 .
$$

The first equality together with the Hermitian conjugate of the second equality $\left[\varepsilon_{\lambda}^{*}-h-\Sigma^{\dagger}\left(\varepsilon_{\lambda}\right)\right]\left|\phi_{\lambda}\right\rangle=0$ gives

$$
\left[h+\frac{1}{2}\left\{\Sigma\left(\varepsilon_{\lambda}\right)+\Sigma^{\dagger}\left(\varepsilon_{\lambda}\right)\right\}\right]\left|\phi_{\lambda}\right\rangle=\operatorname{Re}\left[\varepsilon_{\lambda}\right]\left|\phi_{\lambda}\right\rangle .
$$

That is, we can hermitize the self-energy just like the effective potential in DFT when we solve the QP eigenvalue problem, although the QP energies are complex numbers. It is seen that $\left\{G^{-1}\left(\varepsilon_{\lambda}\right) G^{-1 \dagger}\left(\varepsilon_{\lambda}\right)-G^{-1 \dagger}\left(\varepsilon_{\lambda}\right) G^{-1}\left(\varepsilon_{\lambda}\right)\right\}\left|\phi_{\lambda}\right\rangle=0$, and it should be understood that, inside $\Sigma^{\dagger}\left(\varepsilon_{\lambda}\right)$ and $G^{-1 \dagger}\left(\varepsilon_{\lambda}\right), \varepsilon_{\lambda}$ is replaced by $\varepsilon_{\lambda}^{*}$. The imaginary part of the QP energies is obtained by

$$
\frac{1}{2 i}\left\{\Sigma\left(\varepsilon_{\lambda}\right)-\Sigma^{\dagger}\left(\varepsilon_{\lambda}\right)\right\}\left|\phi_{\lambda}\right\rangle=\operatorname{Im}\left[\varepsilon_{\lambda}\right]\left|\phi_{\lambda}\right\rangle .
$$

Approaching $\omega$ to one of $\varepsilon_{\lambda}$ in Eq. (17) and taking into account Eq. (18), we have $[19,20]$

$$
\begin{aligned}
& \left\langle\phi_{\lambda}\left|\left[1-\left.\frac{\partial \Sigma(\omega)}{\partial \omega}\right|_{\omega=\varepsilon_{\lambda}}\right]\right| \phi_{\lambda}\right\rangle \\
& =\left\langle\phi_{\lambda}\left|\left[1-\left(\left.\frac{\partial \Sigma(\omega)}{\partial \omega}\right|_{\omega=\varepsilon_{\lambda}}\right)^{\dagger}\right]\right| \phi_{\lambda}\right\rangle \\
& =1 .
\end{aligned}
$$

When MBPT calculations do not simultaneously satisfy (20) and (21), one can hermitize the equations like (19). Thus the operator $1-\partial \Sigma(\omega) /\left.\partial \omega\right|_{\omega=\varepsilon_{\lambda}}$ normalizes the QP states. The very important statement here is that this normalization procedure is closely related to the Ward identity $[24,29,33]$

$$
\Gamma_{q=0}(\omega)=1-\frac{\partial \Sigma(\omega)}{\partial \omega},
$$

which is the $q \rightarrow 0$ limit of the Ward-Takahashi identity, where $q \equiv\left(\boldsymbol{q}, \omega^{\prime}-\omega\right)$ denotes the momentum-energy transfer via the Coulomb interaction. In Eq. (22), $\Gamma_{q=0}(\omega)$ is the vertex operator in the $q \rightarrow 0$ limit. Comparing (22) with (21), 
we have

$$
\left\langle\phi_{\lambda}\left|\Gamma_{q=0}\left(\varepsilon_{\lambda}\right)\right| \phi_{\lambda}\right\rangle=1 .
$$

The vertex function, which connects the dynamical interaction to a pair of Green's functions, has an effect to make the system gauge invariant by the Ward-Takahashi identity and guarantees the local charge conservation by the continuity equation [23,24]. However, in the limit $q \rightarrow 0$, no multiple excitation is possible. Therefore the vertex function in this limit counts the $(N \pm 1)$-electron states $\left|\Psi_{\lambda}^{N \pm 1}\right\rangle$ with purely one electron or hole only and has an effect to normalize the corresponding QPWFs to unity, ignoring all the other QPWFs involving multiple excitations. This is the physical meaning of Eq. (23). Now, only for the purpose of the normalization of QP wave functions, we introduce a renormalization function, which satisfies $\sum_{s} \int \phi_{\lambda}^{*}\left(\boldsymbol{r}_{1}, s\right) \Gamma_{q=0 s}^{\mathrm{ren}}\left(\boldsymbol{r}_{1}, \boldsymbol{r}_{2} ; \varepsilon_{\lambda}\right) \phi_{\lambda}\left(\boldsymbol{r}_{2}, s\right) d \boldsymbol{r}_{1} d \boldsymbol{r}_{2}=1$, as

$$
\begin{aligned}
\Gamma_{q=0 s}^{\mathrm{ren}}\left(\boldsymbol{r}_{1}, \boldsymbol{r}_{2} ; \omega\right) & =\left\langle\boldsymbol{r}_{1}, s\left|\Gamma_{q=0}^{\mathrm{ren}}(\omega)\right| \boldsymbol{r}_{2}, s\right\rangle=\delta\left(\boldsymbol{r}_{1}-\boldsymbol{r}_{2}\right) f(\omega), \\
f(\omega) & =\sum_{\lambda \in \text { all }} \frac{1}{\left\langle\phi_{\lambda} \mid \phi_{\lambda}\right\rangle} \frac{\prod_{\alpha \neq \lambda}\left(\omega-\varepsilon_{\alpha}\right)}{\prod_{\beta \neq \lambda}\left(\varepsilon_{\lambda}-\varepsilon_{\beta}\right)} .
\end{aligned}
$$

This is spin-coordinate independent, and $f(\omega)$ satisfies

$$
f\left(\varepsilon_{\lambda}\right)=\frac{1}{\left\langle\phi_{\lambda} \mid \phi_{\lambda}\right\rangle}=\frac{\left\langle\phi_{\lambda}\left|\Gamma_{q=0}\left(\varepsilon_{\lambda}\right)\right| \phi_{\lambda}\right\rangle}{\left\langle\phi_{\lambda} \mid \phi_{\lambda}\right\rangle} .
$$

Then the normalized QP states $\left|\bar{\phi}_{\lambda} ; \varepsilon_{\lambda}\right\rangle=\left|\bar{\phi}_{\lambda}\right\rangle$, which satisfy $\left\langle\bar{\phi}_{\lambda} ; \varepsilon_{\lambda} \mid \bar{\phi}_{\lambda} ; \varepsilon_{\lambda}\right\rangle=1$ but generally obey $\left\langle\bar{\phi}_{\lambda} ; \varepsilon_{\lambda} \mid \bar{\phi}_{\zeta} ; \varepsilon_{\zeta}\right\rangle \neq 0$ for $\lambda \neq \zeta$, can be constructed from the $\omega$-dependent states,

$$
\left|\bar{\phi}_{\lambda} ; \omega\right\rangle=\Gamma_{q=0}^{\mathrm{ren} 1 / 2}(\omega)\left|\phi_{\lambda}\right\rangle=\sqrt{f(\omega)}\left|\phi_{\lambda}\right\rangle .
$$

As well, the normalized Green's function is defined by

$$
\bar{G}(\omega)=\Gamma_{q=0}^{\mathrm{ren} 1 / 2}(\omega) G(\omega) \Gamma_{q=0}^{\mathrm{ren} 1 / 2}(\omega)=G(\omega) f(\omega),
$$

which satisfies $\bar{G}_{s}(1,2)=i D_{s}^{(-)}(1,2)$ for $t_{1}<t_{2}$. That is, $f(\omega)$ becomes $f\left(\varepsilon_{\lambda}\right)$ by taking the pole of the Green's function. It is clear that the normalized QP states satisfy the QP equation,

$$
\varepsilon_{\lambda}\left|\bar{\phi}_{\lambda} ; \varepsilon_{\lambda}\right\rangle=\left[h+\Sigma\left(\varepsilon_{\lambda}\right)\right]\left|\bar{\phi}_{\lambda} ; \varepsilon_{\lambda}\right\rangle
$$

which corresponds to taking the pole of Eq. (27) with (16) in the Dyson equation. The renormalization function $\Gamma_{q=0 s}^{\mathrm{ren}}(1,2)$ connects the original and renormalized vertex functions as

$$
\Gamma_{s}(1,2 ; 3)=\int d 1^{\prime} \Gamma_{q=0 s}^{\mathrm{ren}}\left(1,1^{\prime}\right) \bar{\Gamma}_{s}\left(1^{\prime}, 2 ; 3\right) .
$$

There are two possibilities of choosing $i=1$ or 2 of $\Gamma_{s}(1,2 ; 3)$ to connect $\Gamma_{q=0 s}^{\mathrm{ren}}\left(1,1^{\prime}\right)$ or $\Gamma_{q=0 s}^{\mathrm{ren}}\left(2^{\prime}, 2\right)$. This has to be selected to make the resulting form with $G$ replaced by $\bar{G}$ symmetric. The renormalized vertex function $\bar{\Gamma}$ is the vertex function with factoring out a renormalization function $\Gamma_{q=0 s}^{\mathrm{ren}}\left(i, i^{\prime}\right)$, $\Gamma_{q=0 s}^{\mathrm{ren}}\left(i^{\prime}, i\right)$.

Vertex correction. We will show that changing the Green's function from $G$ to $\bar{G}$ is equivalent to replacing the vertex function $\Gamma$ with $\bar{\Gamma}$ in the exact manner. First, following Baym and Kadanoff [28] and Strinati [24], we separate the two-particle Green's function as $K_{s s^{\prime} ; s s^{\prime}}\left(1,2 ; 1^{\prime}, 2^{\prime}\right)=$ $G_{s}\left(1,1^{\prime}\right) G_{s^{\prime}}\left(2,2^{\prime}\right)-L_{s s^{\prime} ; s s^{\prime}}\left(1,2 ; 1^{\prime}, 2^{\prime}\right)$. Then the first $G G$ term can be included in the one-body term as the Hartree potential $v^{\mathrm{H}}\left(\boldsymbol{r}_{1}\right)$ in Eq. (7). This corresponds

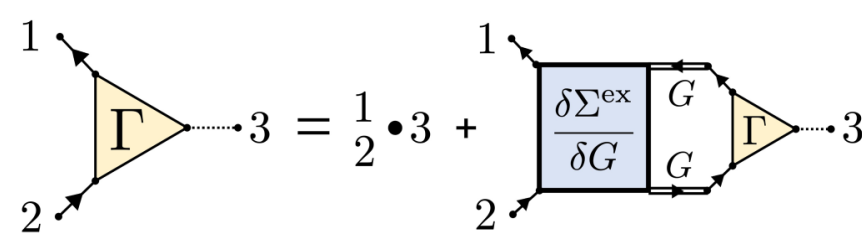

FIG. 2. Diagrammatic representation of the Bethe-Salpeter equation (BSE) for the vertex function $\Gamma_{s}(1,2 ; 3)$.

to separating the self-energy into two terms, $\Sigma_{s}(1,2)=$ $\Sigma^{\mathrm{H}}(1,2)+\Sigma_{s}^{\mathrm{xc}}(1,2)$; the first and second terms represent, respectively, the Hartree term $v^{\mathrm{H}}\left(\boldsymbol{r}_{1}\right) \delta(1,2)$ and the exchange-correlation (xc) term [24]. The density-density correlation function $\chi$ is related to $L$ as [24] $\chi(1,2)=$ $\int d 3 P(1,3) \varepsilon^{-1}(3,2)=-i \sum_{s s^{\prime}} L_{s s^{\prime} ; s s^{\prime}}\left(1,2 ; 1^{+}, 2^{+}\right)$, where $P$ and $\varepsilon^{-1}$ denote, respectively, the polarization function and the inverse of the dielectric function. The polarization function $P(1,3)=-i \sum_{s} R_{s}\left(1,3,1^{+}, 3^{+}\right)$can be calculated from $R_{s}\left(1,3 ; 2,3^{+}\right)=\int d\left(44^{\prime}\right) G_{s}(1,4) G_{s}\left(4^{\prime}, 2\right) \Gamma_{s}\left(4,4^{\prime} ; 3\right)$, where the scalar vertex function satisfies the Bethe-Salpeter equation (BSE) $[1,2,24,33]$ (see also Fig. 2):

$$
\begin{aligned}
\Gamma_{s}(1,2 ; 3)= & \delta(1,3) \delta(2,3)+\sum_{s^{\prime \prime}} \int d\left(44^{\prime} 55^{\prime}\right) \frac{\delta \Sigma_{s}^{\mathrm{xc}}(1,2)}{\delta G_{s^{\prime \prime}}\left(4^{\prime}, 4\right)} \\
& \times G_{s^{\prime \prime}}\left(4^{\prime}, 5\right) G_{s^{\prime \prime}}\left(5^{\prime}, 4\right) \Gamma_{s^{\prime \prime}}\left(5,5^{\prime} ; 3\right) .
\end{aligned}
$$

Then the dielectric function is given by

$$
\varepsilon(1,2)=\delta(1,2)-\int d\left(33^{\prime}\right) \frac{\delta v^{\mathrm{H}}\left(\boldsymbol{r}_{1}\right)}{\delta G_{s^{\prime \prime}}\left(3^{\prime}, 3\right)} R_{s^{\prime}}\left(3^{\prime}, 2 ; 3,2^{+}\right),
$$

and $\sum_{s^{\prime}} L_{s s^{\prime} ; s s^{\prime}}\left(1,3 ; 2,3^{+}\right)=\int d 4 R_{s}\left(1,4 ; 2,4^{+}\right) \varepsilon^{-1}(4,3)=$ $\int d\left(455^{\prime}\right) G_{s}(1,5) G_{s}\left(5^{\prime}, 2\right) \Gamma_{s}\left(5,5^{\prime} ; 4\right) \varepsilon^{-1}(4,3)$. Inserting this expression into (7) and rewriting Eq. (8) as $i \partial_{t_{1}} G_{s}(1,2)-$ $\left[h_{s}\left(\boldsymbol{r}_{1}\right)+v^{\mathrm{H}}\left(\boldsymbol{r}_{1}\right)\right] G_{s}(1,2)-\int d 3 \Sigma_{s}^{\mathrm{xc}}(1,3) G_{s}(3,2)=\delta(1,2)$, we find Hedin's GW $\Gamma$ expression [1,2] for $\Sigma_{s}^{\mathrm{xc}}(1,3)$,

$$
\Sigma_{s}^{\mathrm{xc}}(1,2)=i \int d(34) G_{s}(1,3) W\left(1^{+}, 4\right) \Gamma_{s}(3,2 ; 4),
$$

where $W$ denotes the dynamically screened Coulomb interaction defined by $W(1,2)=\int d 3 \varepsilon^{-1}(1,3) V(3,2)$.

All the above is the exact formulation satisfying the conservation laws $[24,28]$. Now we notice that, although $\Gamma_{q=0 s}(1,2)$ does not have the third argument " 3 ," it satisfies the BSE:

$$
\begin{aligned}
\Gamma_{q=0 s}(1,2)= & \delta(1,2)+\sum_{s^{\prime \prime}} \int d\left(44^{\prime} 55^{\prime}\right) \frac{\delta \Sigma_{s}^{\mathrm{xc}}(1,2)}{\delta G_{s^{\prime \prime}}\left(4^{\prime}, 4\right)} \\
& \times G_{s^{\prime \prime}}\left(4^{\prime}, 5\right) G_{s^{\prime \prime}}\left(5^{\prime}, 4\right) \Gamma_{q=0 s^{\prime \prime}}\left(5,5^{\prime}\right),
\end{aligned}
$$

which has exactly the same form as the BSE for $\Gamma_{s}(1,2 ; 3)$, Eq. (30) (Fig. 2). Equation (33) can be obtained by integrating (30) with respect to "3" because $\int d 3 \Gamma_{s}(1,2 ; 3)=\Gamma_{q=0 s}(1,2) ; \quad$ see, $\quad$ for example, Eq. (7.22) of Ref. [24]. The Ward identity (22) can be easily derived by comparing this BSE with the identities $\partial \Sigma^{\mathrm{xc}}(\omega) / d \omega=\int\left[\delta \Sigma^{\mathrm{xc}}(\omega) / \delta G\left(\omega^{\prime}\right)\right]\left[\partial G\left(\omega^{\prime}\right) / d \omega^{\prime}\right] d \omega^{\prime} \quad$ and $\partial G(\omega) / d \omega=-G(\omega)\left[\partial\left\{\omega-\Sigma^{\mathrm{xc}}(\omega)\right\} / \partial \omega\right] G(\omega)$ in $\omega$ sapce; see, for example, Ref. [33]. Since the $q \rightarrow 0$ limit corresponds to ignoring all multiple excitations, the replacement of 
$\Gamma_{s}(1,2 ; 3)$ with $\Gamma_{q=0 s}(1,2)$ corresponds to treating everything as if there is only one electron or hole in the $(N \pm 1)$-electron states $\left|\Psi_{\lambda}^{N \pm 1}\right\rangle$. Therefore the function associated with its expectation value, $\Gamma_{q=0 s}^{\mathrm{ren}}(1,2)$, has a meaning of the renormalization factor of such a purely one-electron or one-hole QP state as indicated in Eq. (23). The procedure to factor out $\Gamma_{q=0 s}^{\mathrm{ren}}\left(1,1^{\prime}\right)$ or $\Gamma_{q=0 s}^{\mathrm{ren}}\left(2^{\prime}, 2\right)$ symmetrically from $\Gamma_{s}(1,2 ; 3)$ as Eq. (29) is now possible by construction. Then the BSE for the vertex function (30) becomes that for the renormalized one with $\Gamma_{q=0 s}^{\mathrm{ren}}$ factored out:

$$
\begin{aligned}
\bar{\Gamma}_{s}(1,2 ; 3)= & \Gamma_{q=0 s}^{\mathrm{ren}-1}(1,3) \delta(2,3)+\sum_{s^{\prime \prime}} \int d\left(1^{\prime} 44^{\prime} 55^{\prime}\right) \\
& \times \Gamma_{q=0 s}^{\mathrm{ren}-1}\left(1,1^{\prime}\right) \frac{\delta \Sigma_{s}^{\mathrm{xc}}\left(1^{\prime}, 2\right)}{\delta \bar{G}_{s^{\prime \prime}}\left(4^{\prime}, 4\right)} \\
& \times \bar{G}_{s^{\prime \prime}}\left(4^{\prime}, 5\right) \bar{G}_{s^{\prime \prime}}\left(5^{\prime}, 4\right) \bar{\Gamma}_{s^{\prime \prime}}\left(5,5^{\prime} ; 3\right) .
\end{aligned}
$$

Next we find that the exchange-correlation part of the selfenergy (32) becomes

$$
\Sigma_{s}^{\mathrm{xc}}(1,2)=i \int d(34) \bar{G}_{s}(1,3) W\left(1^{+}, 4\right) \bar{\Gamma}_{s}(3,2 ; 4),
$$

and the dielectric function (31) with $R_{s^{\prime}}\left(3^{\prime}, 2 ; 3,2^{+}\right)$becomes

$$
\begin{aligned}
\varepsilon(1,2)= & \delta(1,2)-\int d\left(33^{\prime} 44^{\prime}\right) \frac{\delta v^{\mathrm{H}}\left(\boldsymbol{r}_{1}\right)}{\delta \bar{G}_{s^{\prime \prime}}\left(3^{\prime}, 3\right)} \\
& \times \bar{G}_{s}\left(3^{\prime}, 4\right) \bar{G}_{s}\left(4^{\prime}, 3\right) \bar{\Gamma}_{s}\left(4,4^{\prime} ; 2\right) .
\end{aligned}
$$

Although the calculation of the Hartree potential $v^{\mathrm{H}}\left(\boldsymbol{r}_{1}\right)$ by the original QP wave functions, i.e., the original $G$, was difficult, it is now easy by using the renormalized $\bar{G}$ as

$$
v^{\mathrm{H}}\left(\boldsymbol{r}_{1}\right)=-i \sum_{s} \int d 2 V(1,2) \bar{G}_{s}\left(2,2^{+}\right) .
$$

Then Eq. (36) becomes $\quad \varepsilon(1,2)=\delta(1,2)-$ $\int d 3 V(1,3) P(3,2)$, with the polarization function

$$
P(3,2)=-i \sum_{s} \int d\left(44^{\prime}\right) \bar{G}_{s}(3,4) \bar{G}_{s}\left(4^{\prime}, 3^{+}\right) \bar{\Gamma}_{s}\left(4,4^{\prime} ; 2\right) .
$$

Finally, the dynamically screened Coulomb interaction $W$ and the exchange-correlation part of the self-energy as well as the Hartree potential are completely expressed only by $\bar{G}$ and $\bar{\Gamma}$. This form is no other than the full GW $\Gamma$ formula but with $G$ and $\Gamma$ replaced by $\bar{G}$ and $\bar{\Gamma}$. Thus we succeeded in including the renormalization function (24) in the formulation and established the exact sc GW QPWFs.

In summary, what we have to do is to simply normalize all the QPWFs to unity, although the QP energies are complex. We assumed that the two-time spin-density matrix (or its complementary function) (11) is expressed by $N$ (or infinite) normalized wave functions $\bar{\phi}_{i}(1)$. Then we proved that these functions exactly satisfy the same equation as the time-dependent QP equation (10). Thus the exact equivalence between the normalized wave functions $\bar{\phi}_{i}(1)$ and the QPWFs $\phi_{\mu}(1)$ (except for the normalization factor) was established for the time-dependent case. This is a very reasonable conclusion because each hole (electron) QP state associated with one final PE $\left|\Psi_{\mu}^{N-1}\right\rangle$ (IPE $\left|\Psi_{v}^{N+1}\right\rangle$ ) state should have a norm equal to the amount of one electron exactly. It is clear that the same statement holds for the time-independent case also. If the spin density matrix defined by $\rho_{s}\left(\boldsymbol{r}_{1}, \boldsymbol{r}_{2}\right)=$ $\left\langle\Psi_{0}^{N}\left|\psi_{s}^{\dagger}\left(\boldsymbol{r}_{2}\right) \psi_{s}\left(\boldsymbol{r}_{1}\right)\right| \Psi_{0}^{N}\right\rangle$ is expressed by $N$ normalized wave functions $\bar{\phi}_{i}(\boldsymbol{r})$ as $\rho_{s}\left(\boldsymbol{r}_{1}, \boldsymbol{r}_{2}\right)=\sum_{i}^{N} \bar{\phi}_{i}\left(\boldsymbol{r}_{1}, s\right) \bar{\phi}_{i}^{*}\left(\boldsymbol{r}_{2}, s\right)$, these functions satisfy exactly the same equation as the timeindependent QP equation (9). All these facts imply that one can normalize the QPWFs, although they are not necessarily mutually orthogonal. Here, Baym-Kadanoff conservation laws $[24,28]$ guarantee that we can solve the QP equation with the hermitized self-energy, (19).

We emphasize that to normalize the QPWFs is equivalent to taking into account the Ward identity. The vertex function in the limit $q \rightarrow 0$ counts the $(N \pm 1)$-electron states $\left|\Psi_{\lambda}^{N \pm 1}\right\rangle$ with purely one electron or hole only, and has an effect to normalize the corresponding QPWFs as Eq. (23), ignoring all the other QPWFs involving multiple excitations. Therefore, to normalize the QPWFs is equivalent to replacing $G$ and $\Gamma$, respectively, with $\bar{G}$ normalized by $\Gamma_{q=0}^{\mathrm{ren}}$ and $\bar{\Gamma}$ factored out by $\Gamma_{q=0}^{\text {ren }}$ everywhere. If we assume $\bar{\Gamma}_{s}(1,2 ; 3)=\delta(1,3) \delta(2,3)$, we obtain the renormalized sc GWA, in which $G$ is replaced everywhere by $\bar{G}$. This is not equivalent to the original sc GWA because $\Gamma_{s}(1,2 ; 3)=\Gamma_{q=0 s}^{\mathrm{ren}}(1,3) \delta(2,3)$ is not equal to $\delta(1,3) \delta(2,3)$. The procedure is similar to that proposed by Holm and von Barth [5], but the QP energies should be treated as complex numbers. Although the present theory was explained for the ground state as the initial state, it is applicable to the excited states also by using the approach given by Ohno et al. [34,35].

Finally, we make a comment on the Kohn-Sham formulation of DFT. In their original paper [26] they never wrote that the KS orbitals are mutually orthogonal, although they assumed their normalization. If we accept this interpretation for the KS theory, the present formulation of the sc GW (or sc GW) approach using the normalized QPWFs can be regarded as an extension of the KS theory. Although an indirect relationship between the KS potential and the self-energy has been achieved by Sham and Schlüter [4,27], what we achieved is their direct equivalence in a hermitized form (19), which is exact under the Baym-Kadanoff conservation laws $[24,28]$. The only difference from the traditional DFT is that the QPWFs are not necessarily orthogonal to each other because the self-energy is energy dependent. A nonorthogonality actually implies that the QPWFs are interacting, or correlated, in contrast to the fictitious noninteracting $\mathrm{KS}$ orbitals.

This work was supported by the Grant-in-Aid Scientific Research B program (Grants No. 18H01939 and No. 21H01877) from the Japan Society for the Promotion of Science (JSPS). 
[1] L. Hedin, Phys. Rev. 139, A796 (1965).

[2] L. Hedin and S. Lundqvist, in Solid State Physics, edited by F. Seitz, D. Turnbull, and H. Ehrenreich (Academic Press, New York, 1969), Vol. 23, pp. 1-180.

[3] M. S. Hybertsen and S. G. Louie, Phys. Rev. B 34, 5390 (1986).

[4] R. W. Godby, M. Schlüter, and L. J. Sham, Phys. Rev. B 37, 10159 (1988).

[5] B. Holm and U. von Barth, Phys. Rev. B 57, 2108 (1998).

[6] F. Arywasetiawan and O. Gunnarsson, Rep. Prog. Phys. 61, 237 (1998).

[7] G. Onida, L. Reining, and A. Rubio, Rev. Mod. Phys. 74, 601 (2002).

[8] T. Kotani, M. van Schilfgaarde, and S. V. Faleev, Phys. Rev. B 76, 165106 (2007).

[9] M. Shishkin, M. Marsman, and G. Kresse, Phys. Rev. Lett. 99, 246403 (2007).

[10] F. Caruso, P. Rinke, X. Ren, A. Rubio, and M. Scheffler, Phys. Rev. B 88, 075105 (2013).

[11] P. Koval, D. Foerster, and D. Sánchez-Portal, Phys. Rev. B 89, 155417 (2014).

[12] R. Kuwahara and K. Ohno, Phys. Rev. A 90, 032506 (2014).

[13] M. J. van Setten, F. Caruso, S. Sharifzadeh, X. Ren, M. Scheffler, F. Liu, J. Lischner, L. Lin, J. R. Deslippe, S. G. Louie, C. Yang, F. Weigend, J. B. Neaton, F. Evers, and P. Rinke, J. Chem. Theor. Comp. 11, 5665 (2015).

[14] Y. Takada, Phys. Rev. Lett. 87, 226402 (2001).

[15] A. Grüneis, G. Kresse, Y. Hinuma, and F. Oba, Phys. Rev. Lett. 112, 096401 (2014).

[16] R. Kuwahara, Y. Noguchi, and K. Ohno, Phys. Rev. B 94, 121116(R) (2016).

[17] G. Y. Csanak, H. S. Taylor, and R. Yaris, Adv. Atomic Mol. Phys. 7, 287 (1971).
[18] A. B. Migdal, Sov. Phys. JETP 5, 333 (1957) [Zh. Éksp. Teor. Fiz. 32, 399 (1957)].

[19] A. J. Layzer, Phys. Rev. 129, 897 (1963).

[20] F. Hüser, T. Olsen, and K. S. Thygesen, Phys. Rev. B 87, 235132 (2013).

[21] F. Aryasetiawan, R. Sakuma, and K. Karlsson, Phys. Rev. B 85, 035106 (2012).

[22] Y. Takahashi, Nuovo Cimento 6, 371 (1957).

[23] J. R. Schrieffer, Theory of Superconductivity (Westview Press, Boulder, CO, 1964; CRC Press, Boca Raton, FL, 2018).

[24] G. Strinati, Nuovo Cimento 11, 1 (1988).

[25] P. Hohenberg and W. Kohn, Phys. Rev. 136, B864 (1964).

[26] W. Kohn and L. J. Sham, Phys. Rev. 140, A1133 (1965).

[27] L. J. Sham and M. Schlüter, Phys. Rev. Lett. 51, 1888 (1983).

[28] G. Baym and L. P. Kadanoff, Phys. Rev. 124, 287 (1961).

[29] G. Strinati, H. J. Mattausch, and W. Hanke, Phys. Rev. B 25, 2867 (1982).

[30] A. Klein and R. Prange, Phys. Rev. 112, 994 (1958).

[31] For $i \leqslant N, \widetilde{\phi}_{i}(1, s)$ can be made from $\bar{\phi}_{i}(1, s)$ by using the Gram-Schmidt orthogonalization to all the other $N-1$ orbitals $\bar{\phi}_{j}^{*}(1, s)(j \neq i)$. For $i>N$, an analogous procedure is possible.

[32] This comes from the Dyson equation in two equivalent forms $\quad G(\omega)=G^{0}(\omega)+G^{0}(\omega) \Sigma(\omega) G(\omega)=G^{0}(\omega)+$ $G(\omega) \Sigma(\omega) G^{0}(\omega)$, which corresponds to $\Sigma=\bar{\Sigma}$ in Eq. (6.6) of Ref. [24].

[33] P. Nozières, Theory of Interacting Fermi Systems (Westview Press, 1964; CRC Press, FL, 2018).

[34] K. Ohno, S. Ono, and T. Isobe, J. Chem. Phys. 146, 084108 (2017).

[35] K. Ohno, K. Esfarjani, and Y. Kawazoe, Computational Materials Science: From Ab Initio to Monte Carlo Methods, 2nd ed. (Springer, Berlin, 2018). 